\title{
GGE Biplot and AMMI Analysis of Barley Yield Performance in Iran
}

\author{
B. Vaezi ${ }^{1}$, A. Pour-Aboughadareh ${ }^{2 *}$, R. Mohammadi ${ }^{3}$, M. Armion ${ }^{4}$, A. Mehraban $^{5}$, \\ T. Hossein-Pour ${ }^{6}$ and M. DorII ${ }^{7}$
}

\begin{abstract}
${ }^{1}$ Kohgiluyeh and Boyerahmad Agricultural and Natural Resources Research and Education Center, Agricultural Research, Education and Extension Organization (AREEO), Yasuj, Iran

${ }^{2}$ Department of Crop production and Breeding, Imam Khomeini International University, Qazvin, Iran ${ }^{3}$ Golestan Agricultural and Natural Resources Research and Education Center, Agricultural Research, Education and Extension Organization (AREEO), Golestan, Iran

${ }^{4}$ Ilam Agricultural and Natural Resources Research and Education Center, Agricultural Research, Education and Extension Organization (AREEO), Ilam, Iran

${ }^{5}$ Ardabil Agricultural and Natural Resources Research Center, Agricultural Research, Education and Extension Organization (AREEO), Ardebil, Iran

${ }^{6}$ Lorestan Agricultural and Natural Resources Research and Education Center, Agricultural Research, Education and Extension Organization (AREEO), Lorestan, Iran

${ }^{7}$ Seed and Plant Certification and Registration Institute (SPCRI), Karaj, Iran
\end{abstract}

(Received 7 October 2016; Accepted 19 December 2016; Communicated by T. Harangozó)

Successful production and development of stable and adaptable cultivars only depend on the positive results achieved from the interaction between genotype and environment that consequently has significant effect on breeding strategies. The objectives of this study were to evaluate genotype by environment interactions for grain yield in barley advanced lines and to determine their stability and general adaptability. For these purposes, 18 advanced lines along with two local cultivars were evaluated at five locations (Gachsaran, Lorestan, Ilam, Moghan and Gonbad) during three consecutive years (2012-2015). The results of the AMMI analysis indicated that main effects due to genotype (G), environment (E) and GE interaction as well as four interaction principal component axes were significant, representing differential responses of the lines to the environments and the need for stability analysis. According to AMMI stability parameters, lines G5 and G7 were the most stable lines across environments. Biplot analysis determined two barley mega-environments in Iran. The first mega-environment contained of Ilam and Gonbad locations, where the recommended G13, G19 and G1 produced the highest yields. The second mega-environment comprised of Lorestan, Gachsarn and Moghan locations, where G2, G9, G5 and G7 were the best adapted lines. Our results revealed that lines G5, G7, G9 and G17 are suggested for further inclusion in the breeding program due to its high grain yield, and among them G5 recommended as the most stable lines for variable semi-warm and warm environments. In addition, our results indicated the efficiency of AMMI and GGE biplot techniques for selecting genotypes that are stable, high yielding, and responsive.

Keywords: barley, genotype $\times$ environment interaction, GGE biplot, mega-environment

\footnotetext{
*Corresponding author; E-mail: a.poraboghadareh@edu.ikiu.ac.ir
} 


\section{Introduction}

Barley (Hordeum vulgar L.) is the fourth crop following wheat, rice and maize in the worldwide production of cereals, and growing in arid and semi-arid regions due to its better ability to avoid drought stress than the other crops. The grains of this cereal are used as food and animal fodder, moreover, it has been applied as raw material for the production of beer. Multi-environment trials (MET) play an important role in selecting the best genotypes to be used at different environments. In a MET, a number of genotypes are usually evaluated over several years and locations. The development of genotypes, which can be adapted to a wide range of diverse environments, is the final goal of plant breeders in crop improvement programs (Ahmadi et al. 2015). Genotypes with both high grain yield and stability are identified by growing sets of different genotypes in different environments (Luquez et al. 2002). The two major goals of MET are: (i) to identify locations that best represent the target environments and (ii) to identify the best genotypes/ cultivars for recommendation to farmers. The obtained grain yield for each genotype in each test environment is a measure of the genotype main effect $(\mathrm{G})$, the environment main effect (E), and the genotype-by-environment (GE) interaction (Yan and Kang 2003). Interpretation of performance of a number of genotypes is always affected by large GE interaction. This effect reduces the association between phenotypic and genotypic values and complicates the selection of the best genotypes (Ebdon and Gauch 2002). Hence, modeling the GE interaction in METs helps to determine phenotypic stability of the genotypes for range of locations or a specific genotype for different environmental conditions. Numerous methods have been proposed to analyze the extent of GE interaction under fluctuating growing conditions and determining the adaptation of genotypes. These methods can be classified into three major analytical categories: (i) Univariate parametric analysis, (ii) Non-parametic approaches, and (iii) Multivariate methods for analysis of the GE affects. The additive main effects and multiplicative interaction (AMMI) model is a combines the analysis of variance (ANOVA) with additive factors and the principal component analysis (PCA) with multiplicative factors in a single analysis. Based on the results of AMMI analysis, several stability parameters such as AMMI's stability measures (EVi and EVF), sums of the absolute value of the IPCA scores (SIPCi and SIPCF) and AMMI's stability value (ASV) have been proposed for test of genotypes (Zobel 1994; Purchase 1997; Purchase et al. 2000).

When many genotypes are tested across several years and locations, it is often difficult to determine the pattern of genotypic response across environments without the help of graphical display of the data. The biplot method provides a powerful solution to this problem (Gabriel 1971). Biplot analysis is a multivariate analytical approach that graphically displays the 2-way data and allows visualization of the interrelations among genotypes, environments, and genotypes by environments interactions (Yan and Kang 2003). The GGE biplot methodology contains of a set of biplot interpretation models, whereby important questions regarding genotype evaluation and test-environment evaluation can be visually addressed (Yan et al. 2007). A GGE biplot is formed by plotting the first principal component $(\mathrm{PC})$ scores of the genotypes and the environments against their respective 
scores for the second PC that result from singular value decomposition (SVD) of environment-centered or environment-standardized genotype-by-environment data (GED). This analysis is used to identify high yielding and adapted genotypes as well as suitable test environments (Yan et al. 2000). The best genotypes should have a large PC1 score (high grain yield) and a small PC2 score (high stability). Plant breeders and other agronomists have found GGE biplots useful in test-environment evaluation (Blanche and Myers 2006), genotype evaluation (Kang et al. 2006), mega-environment analysis (Yan and Tinker 2005), trait association and trait profile analyses (Yan and Rajcan 2002), and heterotic pattern analysis (Andio et al. 2004).

Barley is an important field crop in the agricultural system in Iran and usually cultivated in the arid and semi-arid regions (Pour-Aboughadareh et al. 2013). Normal rainfall in these regions is about $<300 \mathrm{~mm}$ which this is one third of average rainfall in the world, while 1.2 percent of the world's land is allocated to Iran (Ahmadi et al. 2016). One way to increase barley production is to grow genotypes best adapted to diverse environments and growing conditions. To evaluate performances of barley, METs are frequently used in Iran. In this regard, several studies on grain yield stability and adaptation have been done on barley in different regions of Iran (Dehghani et al. 2006; Mortazavian et al. 2014; Mohammadi et al. 2015; Khalili and Pour-Aboughadareh 2016), but these studies deal with different plant materials, locations and environments. The objectives of this study were to (i) analyze GE interactions on grain yield of 20 barley lines using AMMI and GGE biplot models (ii) identify barley line (s) that have high yield and stable performance across different environment via AMMI stability statistics and GGE biplot from MET conducted in Iran, and (iii) to determine the best line (s) for each environment as well as discriminate ability and representativeness of the environments.

\section{Materials and Methods}

\section{Multi-environmental barley trials}

Data analyzed in this study were obtained from sets of the national barley multi-environmental yield trials for three years (2013-2015) at five different locations in Iran. The locations consist of Ghachsaran, Moghan, Lorestan, Ilam and Gonbad. More information on the environments is given in Table $\mathrm{S} 1$ * In each environment (location-year combination), 18 advanced lines along with two standard checks were tested. The genotypic code and pedigree of these lines are given in Table S2. In each environment, experimental layout was a randomized complete block design with four replications. Sowing procedure was done by experimental drill in $1.05 \times 7.03 \mathrm{~m}$ plots, consisting of 6 rows with $17.5 \mathrm{~cm}$ row spacing in all experiments. Agriculture practices were optimal for each local agroecological conditions in all tested locations. Fertilizer rate was $50 \mathrm{~kg} \mathrm{ha}^{-1} \mathrm{~N}$ and $75 \mathrm{P}_{2} \mathrm{O}_{5}$ $\mathrm{kg} \mathrm{ha}^{-1}$ applied at planting. Data on grain yield were taken from the middle rows of each plot. At harvest, total grain yield $\left(\mathrm{kg} \mathrm{ha}^{-1}\right)$ was estimated for each genotype at each test environments.

*Further details about the Electronic Supplementary Material (ESM) can be found at the end of the article. 


\section{Statistical analysis}

The AMMI model, which combines standard ANOVA with principal components (PC) analysis (Zobel et al. 1988), was performed to determine the genotype (G), environment (E) and genotype by environment interaction (GE) effects. The AMMI model for the yield of the $i$ th genotype in the $j$ th environment is (Zobel et al. 1988):

$$
Y_{i j}=\mu+g_{i}+e_{j}+\sum_{n=1}^{N} \lambda_{n} \gamma_{i n} \delta_{j n}+\rho_{i j}+\varepsilon_{i j}
$$

where $\mu$ is the grand mean; $g_{i}$ is the main effect of the $i$ th genotype $(\mathrm{G}) ; e_{j}$ is the main effect of the $j$ th environment (E);

$$
\sum_{n=1}^{N} \lambda_{n} \gamma_{i n} \delta_{j n}+\rho_{i j}+\varepsilon_{i j}
$$

is the GE interaction, where $\lambda_{n}$ is the eigenvalue of the $n$th interaction principal component analysis (IPCA) retained in the AMMI model; $\gamma_{i n}$ is the eigenvector for the $i$ th genotype from $n$th IPCA, $\delta_{j n}$ is the eigenvector for the $j$ th environment from the $n$th IPCA, $\rho_{i j}$ is the GE interaction residual, and $\mathrm{N}$ is the number of IPCA retained in the model, and $\varepsilon_{i j}$ is the random error term. Based on the results of AMMI analysis, several parametric stability statistics including the AMMI's stability measures (EV1 and EVF), sums of the absolute value of the IPCA scores (SIPC1 and SIPCF), AMMI statistic coefficient (Di and DF), and AMMI's stability value (ASV) were calculated using the formulas proposed by Zobel (1994), Zhang et al. (1998) and Purchase et al. (2000).

After detecting the GE interaction, the data were graphically analyzed to interpret stability and adaptability using the GGE biplot software (Yan et al. 2000). The GGE biplot methodology is composed of the biplot (Gabriel 1971) and the GGE (Yan et al. 2000) concepts. The detailed description of the principal of GGE-biplot can be found in the review of Yan and Tinker (2006). The results obtained from this analysis are displayed three ways $(i)$ graphical view exhibiting concentric circles with vectors of entries that shows information about associations among environments and genotypes to identify stable genotype, (ii) polygon view a biplot to interpret adaptability of the genotypes in a particular environment and (ii) identify the ideal genotype base on both mean and stability.

\section{Results}

\section{AMMI model}

The results of AMMI analysis of grain yield data for eighteen advanced lines along with two check cultivars and 15 environments are presented in Table 1 . The results indicated that $73.94 \%$ of the total variation was attributable to environmental effects, $1.28 \%$ to genotypic effects, and $7.71 \%$ to GE interaction effects. Furthermore, results showed values for the first four principal components (IPCA) were also highly significant. The four IPCAs of GE interaction accounted for $71.71 \%$ of the total variation effect it had on the 
Table 1. AMMI analysis on grain yield of the 20 barley lines across 15 environments

\begin{tabular}{|l|c|c|c|c|}
\hline Source of variation & df & MS & $\%$ TTS & $\%$ GE \\
\hline Total & 1199 & 1998433.51 & & \\
\hline Treatment & 299 & $6647234.30^{* *}$ & 82.94 & \\
\hline Genotype (G) & 19 & $1620760.58^{* *}$ & 1.28 & \\
\hline Environment (E) & 14 & $126555126^{* *}$ & 73.94 & \\
\hline Block & 45 & $2000888.87^{* *}$ & 3.75 & \\
\hline G×E interaction & 266 & $695326.47^{* *}$ & 7.71 & \\
\hline IPCA1 & 32 & $1557061.81 * *$ & 2.07 & 26.93 \\
\hline IPCA2 & 30 & $1200180.93 * *$ & 1.50 & 19.46 \\
\hline IPCA3 & 28 & $1057939.29 * *$ & 1.23 & 16.01 \\
\hline IPCA4 & 26 & $661241.80 *$ & 0.71 & 9.29 \\
\hline Residual & 150 & 348739 & 2.18 & 28.28 \\
\hline Error & 855 & 372583.30 & 13.29 & 172.23 \\
\hline
\end{tabular}

TSS and GE indicate Total Sum of Squares and Genotype-by-environment interaction, respectively. $*$ and ${ }^{* *}$ indicate significant at the 5 and $1 \%$ levels, respectively.

variation of grain yield. The first IPCA1 explained $26.94 \%$ of the variation affected by interaction, while IPCA2, IPCA3 and IPCA4 accounted for 19.47, 16.01 and 9.29\%, respectively. The GE interaction effect was greater by six times than the genotype effect showing the presence of remarkable interactions (Table 1). Hence, this is supported by the fact that the GE mean grain yield varied from $1278.70 \mathrm{~kg} \mathrm{ha}^{-1}$ (corresponding to G6 at Ilam) to $5007 \mathrm{~kg} \mathrm{ha}^{-1}$ (corresponding to G1 at Gachsaran). The average yield per location also varied from 2187.59 , in Ilam to $3898.12 \mathrm{~kg} \mathrm{ha}^{-1}$ in Gachsaran. The mean grain yield of the tested lines varied from 1860.79 to $2305.53 \mathrm{~kg} \mathrm{ha}^{-1}$, with an average of $2091.54 \mathrm{~kg}$ $\mathrm{ha}^{-1}$ (Table 2). The line G9 followed by lines G2 and G5 had the highest average yield, while the lines G6, G8 and G10 had poor performance.

The AMMI-based stability parameters calculated based on four IPCAs which were retained in the AMMI model via $F$-test (Table 2). In the case of EV1, line G10 was the most stable line followed by G5, G7, G20 and G14, whereas G13, G6, G19, G11 and G3 had highest values of EV1. The EVF statistic ranked lines G7, G17, G20, G5 and G1 as the five most stable lines and G6, G9, G18, G13 and G10 as the five unstable ones (Table 2). The first five stable lines ranked in the top based on the SIPC1 parameter were G10, G5, G7, G20 and G14; and based on SIPCF parameter the most stable line was G7, followed by G1, G8, G17 and G20. The AMMI stability value (ASV), which uses the first two principal components scores (PCA) to produce a balanced measurement between them, and can be useful in situations where the two first IPCs explained considerable amount of genotype by environment interactions. According to this parameter, lines G5, G7, G4, G10 and G14 which had lower values of ASV, identified as stable lines, but lines G6, G13, G8, G19 and G11 identified as more unstable lines. In this case, G5 with ranking 


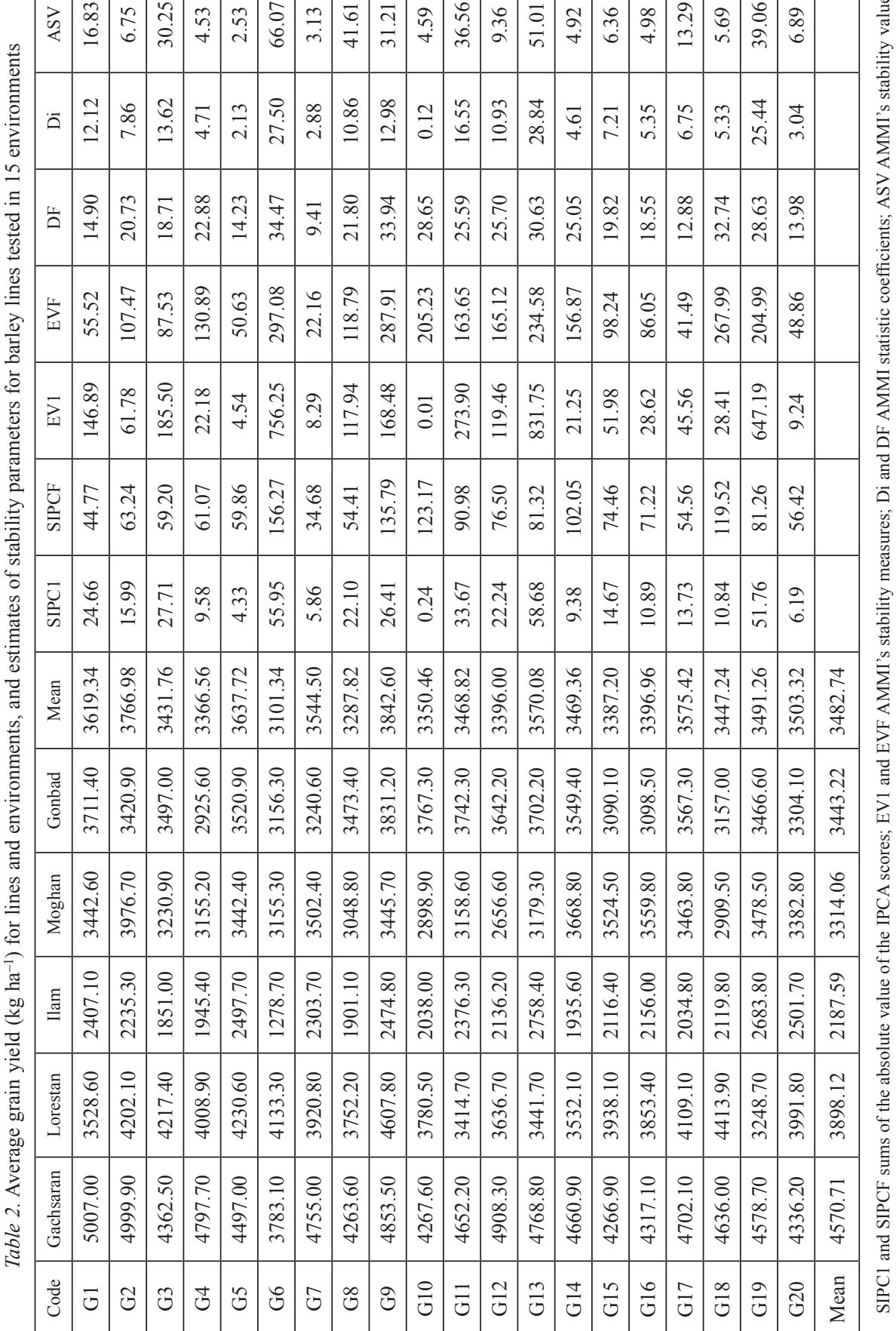

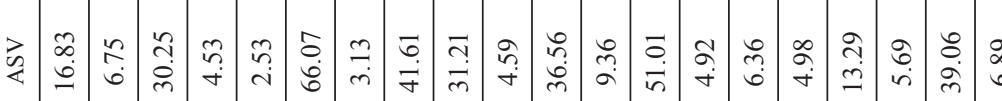
乎 产 
of 3 in mean performance had higher stability. According to D1 parameter, the lines G10, G5, G7, G20 and G14 had the lowest values, whereas the highest values observed for G13 followed by G6, G18, G11 and G3. According to DF stability statistic, line G7, followed by G17, G20, G5 and G1 were better in terms of grain yield and adaptability, while G6, G9, G18, G13 and G10 with higher values were the worst ones.

\section{GGE biplot methodology}

The GGE biplot analysis was used to identify the best line at each environment and assess the stability of the lines. The biplot analysis gave a good visual evaluation of GE based on grain yield which explained $44.7 \%(\mathrm{PC} 1=25.7$ and $\mathrm{PC} 2=19 \%)$ of the total variation across the test environments (Fig. 1A). Discriminant test environment perfectly resolve genotype differences, so providing the necessary information for selection by a breeder. Based on results, the environmental vector biplot identified E6, E7, E8, E9 and E14 as highly discriminating ability for the lines tested, as evidenced by the large environment vectors. The vector view of the GGE biplot also indicated the interrelationships between environments (Fig. 1A). The angle between the vectors of two environments estimates the correlation between them. Therefore, environments E7, E9 (Lorestan location), E4 and E6 (Moghan location) were highly correlated and nearly identical in their ability to discriminate among genotypes for yield performance (particularly for G2 and G9). The maximum angle among the vectors corresponding to Ilam (E13, E14 and E15), Gachsaran (E1, E2 and E3) and Gonbad (E10, E11 and E12) is below 90 ${ }^{\circ}$, indicating that these locations discriminate genotypes in a similar fashion. The environment E8 corresponding to Lorestan location made an obtuse angle. This suggests that this environment tend to be distinctly independent.

To explore the possible existence of mega-environments within the locations, a scatter plot with polygon bisectors was constructed to visualize the interaction patterns between lines and the test locations. Thus, based on biplot analysis of 3-year of data, six sectors with two mega-environments with different "winning" lines were identified (Fig. 1B). The vertex lines were G2, G9, G13, G11, G10 and G6. The vertex line in each sector shows the highest yielding line in the environment that fell within that specific sector (Yan et al. 2000). Also, the first mega-environment contains locations Gonbad (E4) and Ilam (E5), with line G13 being the winner and the second mega-environments contains locations Gachsaran (E1), Moghan (E2) and Lorestan (E3) with line G2 and G9 being the winner. The rest of lines were contained within the polygon and had shorter vectors, proposing that they were relatively less responsive to the interaction with the environments.

The mean yield and stability performance view biplot (Fig. 1C) was used to consider stability of the 20 barley lines across the five environments (average of 3 -year data). This biplot accounted for $44.70 \%$ of the total variation in grain yield. In this biplot, average environment axis or the axis of the average environment coordinate (AEC) abscissa is the single-arrowed line that passes through the average environment and biplot origin. The axis of the AEC ordinate is the double-arrowed line that passes through the biplot origin and is perpendicular to the AEC abscissa. The genotypes were ranked along the average 

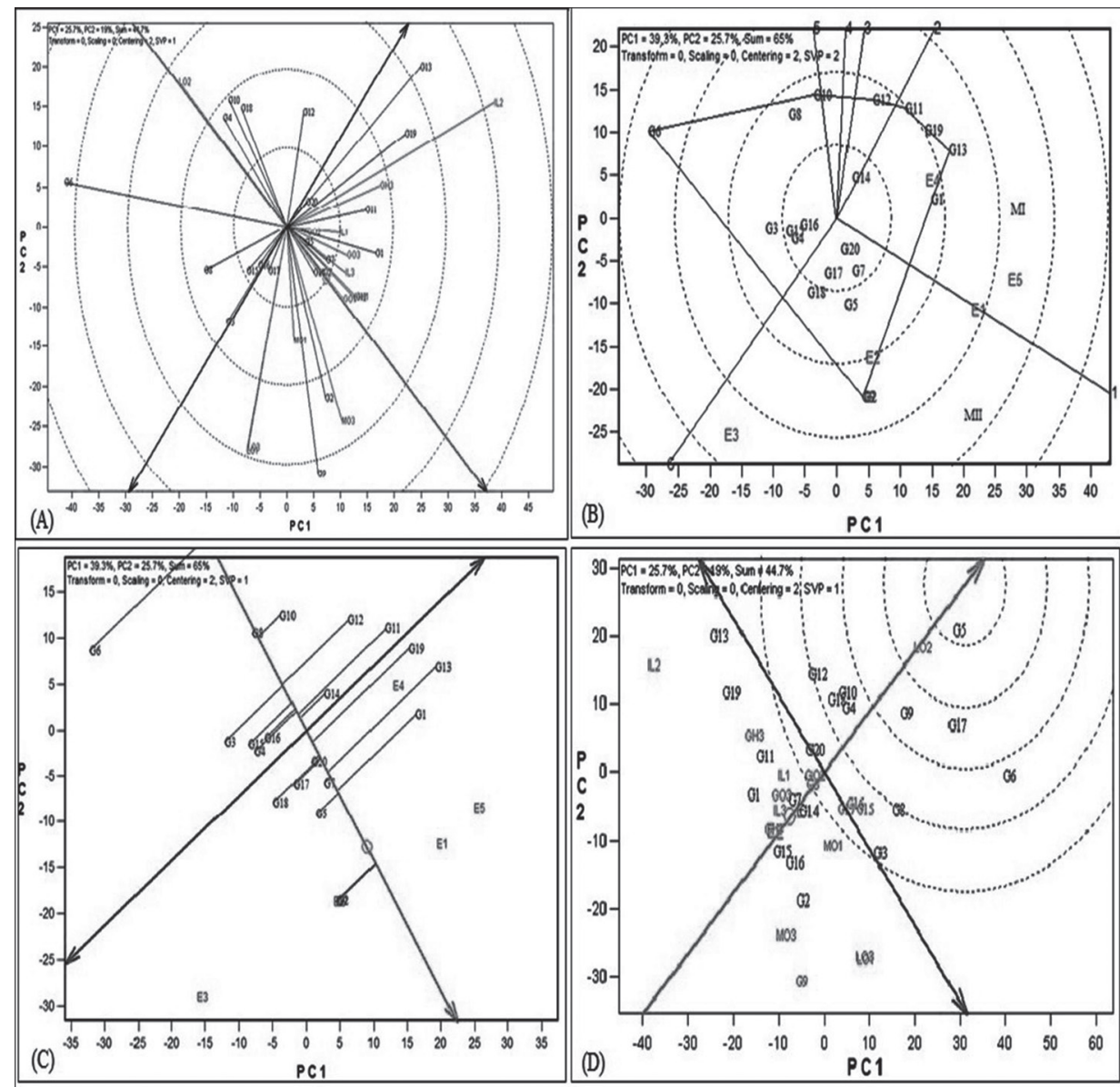

Figure 1. (A) The environment vector bi-plot showing environmental differences in discriminating the 20 barley advanced lines for grain yield at the 15 test environments, (B) An environment focused bi-plot showing "winning" lines for the two different mega environments for grain yield at the five locations, (C) The mean versus stability view of the genotype main effect plus genotype $\times$ environment interaction biplot, and (D) Comparison of barley lines against the 'ideal' genotype for grain yield and stability of performance across environments. E1, E2, E3, E4 and E5 indicate Gachsaran, Moghan, Lorestan, Ilam and Gonbad, respectively. GH1-3, MO1-3, LO1-3, GO1-3 and IL1-3 indicate E1-3, E4-6, E7-9, E10-12 and E13-15, respectively (See Table S1)

environment axis, with the arrow pointing to the highest value based on mean performance across all environments (Yan et al. 2007). On the basis of the projection of each line onto the average environment axis, the nine top lines were ranked as G2 $>$ G9 $>$ G5 $>$ $\mathrm{G} 1>\mathrm{G} 7>\mathrm{G} 13>\mathrm{G} 20>\mathrm{G} 17>\mathrm{G} 18>\mathrm{G} 5$. Furthermore, the stability of the line was measured due to their projections onto the AEC ordinates (Yan et al. 2007). Three lines G5, G17 and G7 were the most stable because they had short projection onto AEC ordinate. 
Among the stable lines, G5 was the high-yielding performance across all environments. The most high-yielding G9, was not among the most stable lines, suggesting that this line may be have specific adaptation to some of the environment. Also, the local checks, G1 and G2, with high-yielding performance were relatively stable. An ideal genotype is one that has both high mean grain yield and high stability. Hence, Fig. 1D revealed that the line G5 was the closet to the ideal genotype, hence this genotype seems to be widely adapted across several environments.

\section{Discussion}

In the present study, AMMI model and GGE biplot analysis allowed a significant and useful summary of GE interactions and helped in examining the relationships as well as variations in genotype performance among various testing environments. The AMMI analysis showed that the main effects due to genotype $(\mathrm{G})$, environment $(\mathrm{E})$ and GE interaction were highly significant (Table 1). A large variation explained by environments showed that the environments were diverse with large differences among environmental means causing most of the variation in grain yield (Shukla et al. 2015). The small portion of the total sum of square due to treatment was attributed to genotypic $(\mathrm{G})$ effect whereas, the magnitude of GE interaction sum of squares was higher than $G$ effect revealed that there were considerable differences in genotypic response across environments (Shukla et al. 2015). Simultaneous consideration of the AMMI-based stability parameters for the individual lines (Table 2) indicated that parameters which use the first of IPCA (containing the SPICi1, Di1 and EVi1) gave similar ranking patterns, indicating that any of these parameters would be suitable for selecting desirable genotypes. Hence, according to all AMMI's stability parameters, lines G5 and G7 identified as stable lines than other lines. Similarly, Jamshidmoghaddam and Pourdad (2011) found the same ability of stability statistics derived from the first IPC of AMMI in safflower MET. Mohammadi and Amri (2013) also used AMMI's stability parameters to screening of durum wheat, so that they reported satisfactory ability of these parameters in identification of stable genotypes. Also, they are denoted that the stability parameters which use number of IPCA retained in the AMMI model (i.e. SIPCF, DF and EVF) are better for stability analysis than those use the first of IPCA.

GGE biplot analysis provided a framework for classifying the target testing locations that differentiates between genotypes that are high yielding and stable. If genotype effect (G) is sizable, PC1 scores will be highly correlated with G and PC2 will be controlled by GE interaction and (Yan and Tinker 2005). In this study, G7, G17, G5 and G20 with intermediate grain yield and high yield stability were identified as the best lines. In addition, these lines have a large PC1 score and a small PC2 score (Fig. 1A). On the other hand, the lines G6, G13 and G19 were the best in some of the environments because they had the longest distance from the origin of the biplot (Yan et al. 2007). The GGE biplot also provide a good tool for consideration of interrelationships among all environments. Yan and Rajcan (2002) indicated that the GGE biplot describes the interrelationships among all environments on the basis of overall pattern of the MET data whereas simple correlation 
coefficients only describe the relationships between two environments. The angle between the environmental vectors shows the correlation coefficient between them. In this research, the majority of the correlations among Ilam, Gonbad and Gachsaran environments were high and positively significant (Fig. 1A), suggesting that these environments are very similar. Yan (2001) reported that the 'which-won-where' pattern is another important graphical pattern for studying the MET data for identification of different megaenvironments. In fact, the polygon view of GGE biplot is the best technique for recognizing the best genotype in each mega-environment. The polygon plot (Fig. 1B) in the present study also identified two mega-environments for barley in Iran. This result had several implications for future breeding and genotypes evaluation of barley in Iran. First, promising barley lines should be deployed for the two mega-environments to achieve best adaptation. Second, the fact that two mega-environments were identified suggests that specific adaptation could be positively exploited (Ceccarelli 1996). For instance, according to Fig. 1B, high-yielding lines in each of the mega-environments are different, which means specific adaptation of a line to a mega-environment and positive utilization of the GE interaction. This result reveals that some of lines, particularly the line G5 followed by G7, G17 and G20 from ICARDA are more adapted to different environments of Iran. Also, based on results of this study there was two excellent locations for M-I, and three main locations for M-II. This is an important result from our study, with implications for future barley breeding programs in Iran.

Use of GGE biplot analysis gave us good visual information on genotype performance and stability. According to described recommendation by Yan and Tinker (2006), an ideal genotype should have both high mean yield and high stability within a mega-environment. The most stable test barley advanced lines along with the local checks in the present study were G2, G9, G5, G1, G7, G13, G20, G17, G18 and G5 because their short projection onto the AEC ordinate (Fig. 1C). Among the most stable lines, G5, G7 and G17 had the highest yield across all the 15 environments. As shown in Fig. 1D, the line G5 is included in the first inner cycle, suggesting that it could be a superior line for warm and semi-warm regions of Iran. The other highest yielding G9 and G17 was not among the most stable, suggesting that these lines may have specific adaptation to some of the environments as previously reported by Dehghani et al. (2006) who identified high yielding but unstable barley genotype in divers-environments of Iran. The local checks (G1 and G2) were among the highest yielding lines in this study but not very stable in the test environments. Therefore, the line G5 with acceptable grain yield and stable across environments should be recommended for future breeding programs related to cultivar release in warm and semi-warm regions of Iran and other similar environments for adoption.

Selection of superior lines/cultivars for stability is required in rainfed conditions, where the environment is changeable and unpredictable. Therefore, genotype assessment under variable environments and adoption of simultaneous selection for grain yield and yield stability is the most valuable selection parameter that can lead to desirable genotypes. In conclusion, the important results obtained from this research could be listed follows: 
(i) The genotype (G) and environment (E) main effects and GE interaction effect were significant for barley advanced lines tested in warm and semi-warm regions of Iran.

(ii) The lines G5, G7 and G17 are suggested for further inclusion in the breeding program due to its high grain yield, and G5 is the most recommended for release in barleygrowing areas of Iran because of it showed the most stability and high grain yield.

(iii) In addition to commonly and standard used data analysis techniques, AMMI analysis and biplot methodology offer additional features, preferably in the part of graphical displaying and understanding of important interactions which are omnipresent in the datasets from crop science research.

\section{Acknowledgements}

This research was supported by grant 03-56-1508-92164 from the Agricultural and Natural Resources Research and Education Center, Agricultural Research, Education and Extension Organization (AREEO), Iran. We would like to thank all members of the project who contributed to the implementation of the field work.

\section{References}

Ahmadi, J., Vaezi, B., Shaabani, A., Khademi, K., Fabriki-Ourang, S., Pour-Aboughadareh, A. 2015. Nonparametric measures for yield stability in grass pea (Lathyrus sativus L.) advanced lines in semi warm regions. J. Agr. Sci. Tech. 17:1825-1838.

Ahmadi, J., Vaezi, B., Pour-Aboughadareh, A. 2016. Analysis of variability, heritability, and interrelationships among grain yield and related characters in barley advanced lines. Genetika 48:73-85.

Andio, F.L., Cravero, V., Asprelli, P., Firpo, T., Garcia, S.M., Cointry, E. 2004. Heterotic patterns in hybrids involving cultivar groups of summer squash. Euphytica 135:355-360.

Blanche, S.B., Myers, G.O. 2006. Identifying discriminating locations for cultivar selection in Louisiana. Crop. Sci. 46:946-949.

Ceccarelli, S. 1996. Positive interpretation of genotype by environment interactions in relation to sustainability and biodiversity. In: Cooper, M., Hammer, G.L. (eds), Plant Adaptation and Crop Improvement. Wallingford, UK. pp. 467-486.

Dehghani, H., Ebadi, A., Yousefi, A. 2006. Biplot analysis of genotype by environment interaction for barley yield in Iran. Agron. J. 98:388-393.

Ebdon, J.S., Gauch, H.G. 2002. Additive main effect and multiplicative interaction analysis of national turf grass performance trials: I. Interpretation of genotype environment interaction. Crop. Sci. 42:489-496.

Gabriel, K.R. 1971. The biplot graphic display of matrices with application to principal component analysis. Biometrika 58:453-467.

Jamshidmoghaddam, M., Pourdad, S.S. 2011. Genotype $\times$ environment interactions and simultaneous selection for high oil yield and stability in rainfed warm areas rapeseed (Brassica napus L.) from Iran. Euphytica 180:321-335.

Kang, M.S., Aggarwal, V.D., Chirwa, R.M. 2006. Adaptability and stability of bean cultivars as determined via yield-stability statistic and gge biplot analysis. J. Crop. Improv. 15:97-120.

Khalili, M., Pour-Aboughadareh, A. 2016. Parametric and non-parametric measures for evaluating yield stability and adaptability in barley doubled haploid Lines. J. Agr. Sci. Tech. 18:789-803.

Luquez, J.E., Aguirrezabal, L.A.N., Aguero, M.E., Pereyra, V.R. 2002. Stability and adaptability of cultivars in non-balanced yield trials: Comparison of methods for selecting high oleic sunflower hybrids for grain yield and quality. J. Agron. Crop. Sci. 188:225-234. 
Mohammadi, M., Noorinia, A.A., Khalilzadeh, G.R., Hosseinpoor, T. 2015. Application of GGE biplot analysis to investigate GE interaction on barley grain yield. Curr. Opin. Agric. 4:25-32.

Mohammadi, R., Amri, A. 2013. Genotype $\times$ environment interaction and genetic improvement for yield and yield stability of rainfed durum wheat in Iran. Euphytica 192:227-249.

Mortazavian, S.M.M., Nikkhah, H.R., Hassani, F.A., Hosseini, S., Taheri, M., Mahloohi, M. 2014. GGE biplot and AMMI analysis of yield performance of barley genotypes across different environments in Iran. J. Agr. Sci. Tech. 16:609-622.

Pour-Aboughadareh, A., Naghavi, M.R., Khalili, M. 2013. Water deficit stress tolerance in some of barley genotypes and landraces under field conditions. Not. Sci. Biol. 5:249-255.

Purchase, J.L. 1997. Parametric analysis to describe GE interaction and yield stability in winter wheat. PhD thesis. Department of Agronomy, Faculty of Agriculture, University of the Orange Free State. Bloemfontein, South Africa.

Purchase, J.L., Hatting, H., Van Deventer, C.S. 2000. Genotype $\times$ environment interaction of winter wheat in south africa: II. Stability analysis of yield Performance. S. Afr. J. Plant. Soil. 17:101-107.

Shukla, S., Mirshra, B.K., Siddiqui, A., Pandey, R., Rastogi, A. 2015. Comparative study for stability and adaptability through different models in developed high the baine lines of opium poppy (Papaver somniferum L.). Ind. Crop. Prod. 74:875-886.

Yan, W., Hunt, L.A., Sheng, Q., Szlavnics, Z. 2000. Cultivar evaluation and mega-environment investigation based on the GGE biplot. Crop. Sci. 40:597-605.

Yan, W. 2001. GGE biplot a windows application for graphical analysis of multi-environment trial data and other types of two-way data. Agron. J. 93:1111-1118.

Yan, W., Rajcan, I. 2002. Biplot evaluation of test sites and trait relations of soybean in Ontario. Crop Sci. 42:11-20.

Yan, W., Kang, M.S. 2003. GGE Biplot Analysis: a Graphical Tool for Breeders, Geneticists, and Agronomists. CRC Press. Boca Raton, FL, USA.

Yan, W., Tinker, N.A. 2005. An integrated system of biplot analysis for displaying, interpreting, and exploring genotype by environment interactions. Crop. Sci. 45:1004-1016.

Yan, W. and Tinker, N.A. 2006. Biplot analysis of multi-environment trial data: principles and applications. Can. J. Plant. Sci. 86:623-645.

Yan, W., Kang, M.S., Ma, B., Woods, S., Cornelius, P. 2007. GGE Biplot vs. AMMI analysis of genotype by environment data. Crop. Sci. 47:643-655.

Zhang, Z., Lu, C., Xiang, Z.H. 1998. Stability analysis for varieties by AMMI model. Acta Agron. Sin. 24:304-309.

Zobel, R.W. 1994. Stress resistance and root systems. In: Proc. of the Workshop on Adaptation of Plant to Soil Stress. Institute of Agriculture and Natural Resources University of Nebraska, Lincoln, INTSORMIL Publ. Lincoln, NE, USA. pp. 80-99.

Zobel, R.W., Wright, M.J., Gauch, H.G. 1988. Statistical analysis of a yield trials. Agron. J. 80:388-393.

\section{Electronic Supplementary Material (ESM)}

Electronic Supplementary Material (ESM) associated with this article can be found at the website of CRC at http://www.akademiai.com/content/120427/

Electronic Supplementary Table S1. Agro-climatic characteristics of environments in yields stability experiments for 20 barley lines

Electronic Supplementary Table S2. Genotypic code, name and origin of the tested barley lines 